Original Paper http://ajol.info/index.php/ijbcs http://indexmedicus.afro.who.int

\title{
Pratiques et contraintes de l'élevage de la chèvre rousse de Maradi en milieu rural au Niger
}

\author{
M'Bareck MOLID AZIADA $^{1 *}$, Ousseini MAHAMAN M. MOUCTARI ${ }^{2}$, Salissou ISSA $^{1}$ \\ et Mahamadou CHAIBOU ${ }^{2}$
}

${ }^{1}$ Département des Productions Animales, Institut National de la Recherche Agronomique du Niger, BP 429, Niamey, Niger.

${ }^{2}$ Département des Productions Animales, Faculté d'Agronomie, Université Abdou Moumouni de Niamey, BP 10960, Niamey, Niger.

*Auteur correspondant ; E-mail : molidmbareck@gmail.com; Tel. +22790387584

\section{REMERCIEMENTS}

Les auteurs remercient le Projet Recherche Développement pour la Sécurité Alimentaire et l'Adoption au Changement Climatique (RED-SAACC) qui a financé entièrement les travaux de recherche.

\begin{tabular}{lll}
\hline Received: 30-03-2021 & Accepted: 16-06-2021 & Published: 30-06-2021 \\
\hline
\end{tabular}

\section{RESUME}

Au Niger, l'élevage caprin occupe une place importante dans l'économie des ménages ruraux. Une étude a été conduite dans la région de Maradi afin de caractériser les pratiques de gestion des élevages des chèvres rousses et analyser leurs contraintes. Ainsi, 180 agro-éleveurs répartis dans 12 villages de la région ont été questionnés. Les thèmes abordés au cours des interviews ont porté sur : les caractéristiques du ménage, les objectifs de production, le mode de conduite des animaux, la gestion de l'alimentation, la santé animale ainsi que les contraintes auxquelles les producteurs font face. Les résultats ont révélé que la majorité $(72,8 \%)$ des propriétaires des caprins sont des femmes. Les caprins sont élevés en priorité comme épargne pour subvenir aux besoins du ménage. Pendant la saison sèche, plus de la moitié (51\%) des éleveurs font recours à l'achat du son de blé pour la complémentation des animaux. Diverses sources des compléments minéraux sont utilisées, notamment le sel de cuisine, les pierres à lécher, le natron et le sel de Bilma. La pénurie d'aliments a été identifiée comme la contrainte majeure de l'élevage caprin (57,8\%). Elle est suivie par les maladies animales $(17,2 \%)$ et la stabulation en saison de pluies $(7,8 \%)$. L'amélioration de l'élevage de la chèvre rousse passe par la prise en compte de toutes les contraintes de production.

(C) 2021 International Formulae Group. All rights reserved.

Mots clés : Chèvre rousse, élevage contraintes, Maradi, Niger.

\section{Practices and constraints of Maradi red goat breeding in rural areas in Niger}

\begin{abstract}
In Niger, goat farming plays an important role in the economy of rural households. A study was conducted in the Maradi region to characterize the management practices of red goat farms and analyze their constraints. Thus, 180 agro-pastoralists spread out in 12 villages of the region were questioned. The themes
\end{abstract}


addressed during the interviews included: household characteristics, production objectives, animal husbandry, feed management, animal health and the constraints faced by producers. The results revealed that the majority $(72,8 \%)$ of goat owners were women. The feeding system is based on natural pastures and crop residues. During the dry season, more than half $(51 \%)$ of the farmers used wheat bran for animal complementation. Various sources of mineral supplements are used, including cooking salt, lickstones, natron and Bilma salt. Food shortage has been identified as the major constraint to goat farming $(57.8 \%)$, followed by animal diseases $(17.2 \%)$ and stalling during the rainy season $(7.8 \%)$. The improvement of the breeding of the red goat requires the taking into account of all the constraints of production.

(C) 2021 International Formulae Group. All rights reserved.

Keywords: Red goat, practices, breeding constraints, Maradi, Niger.

\section{INTRODUCTION}

L'élevage des caprins contribue à la réduction de la pauvreté et à l'amélioration significative de l'alimentation des populations (FAO, 2013). Au Niger, on estime l'effectif du cheptel à environ 14 millions d'Unité Bétail Tropical (UBT) (Laouali, 2014) dont 36, 21\% sont des caprins (FAO stat, 2017). Deux principales races des caprins sont distinguées: la chèvre du sahel majoritaire qu'on rencontre dans toutes les zones agroécologiques, et la chèvre rousse de Maradi élevée dans le centre-sud du pays. La région de Maradi est le berceau de la chèvre rousse qui compte près de $60 \%$ des effectifs caprins roux du Niger. Malgré que son chef-lieu de région soit considéré comme la capitale économique du Niger, la région de Maradi est classée la plus pauvre du pays. Selon les résultats de l'enquête de l'Institut National de la Statistique (INS) sur le profil de pauvreté, $79,7 \%$ de la population de cette région est pauvre, soit près de 8 personnes sur 10 (INS, 2005). A Maradi, depuis près de 10 ans, les taux de malnutrition aigüe ou chronique sont les plus élevés du pays (FAO, 2015). Dans un tel contexte, l'élevage de la chèvre rousse participe à l'amélioration des moyens d'existence des populations rurales. Cependant, cet élevage fait face à des difficultés liées principalement aux problèmes d'alimentation. En effet, les systèmes d'élevage caprin reposent généralement sur l'utilisation des ressources des parcours naturels, souvent très pauvres et sujets à d'importantes variations saisonnières (FAO, 2015). Une mauvaise alimentation entraînerait une chute de production de lait et des performances de reproduction faibles, une croissance lente, une perte de l'état corporel et une sensibilité accrue aux maladies (Aduguna et al., 2000). Plusieurs études ont été menées sur la chèvre rousse de Maradi, notamment sur sa caractérisation phénotypique et zootechnique et son alimentation en milieu contrôlé (Marichatou et al., 2002 ; Harouna, 2013 ; Boureima, 2015 ; Mahamadou et al., 2017 ; Nourou et al., 2018; Barazi et al., 2019). Néanmoins, les performances de production restent en deçà des attentes des producteurs. L'amélioration des systèmes d'alimentation passe par la prise en compte des pratiques des éleveurs et des contraintes auxquelles ils font face. L'objectif de ce travail est d'analyser les pratiques de gestion des élevages des chèvres rousses par les producteurs afin d'identifier les contraintes auxquelles ils sont confrontés.

\section{MATERIEL ET METHODES Zone d'étude}

L'étude a été menée dans la région de Maradi de juillet à septembre 2018. Cette région est située dans la partie centre sud du Niger (Figure 1). Sur la base des précipitations annuelles reçues on distingue trois (3) zones agro-écologiques: la zone sahélo-saharienne, la zone sahélienne et la zone sahélo-soudanienne. Maradi est considérée comme le poumon économique du Niger grâce à sa proximité avec la République Fédérale du Nigéria à travers d'importants échanges commerciaux. Elle est la deuxième région la plus peuplée du pays avec une population estimée à 3404645 habitants en 2012 (INS, 2015) et occupe seulement 3\% de la superficie du pays. Plus de $80 \%$ de cette population vie en milieu rural et pratique 
l'agriculture et l'élevage. Elle est alors la région agricole par excellence et est la première productrice des cultures pluviales du Niger à savoir le mil, le sorgho et le maïs auxquels s'ajoutent l'arachide et le sésame comme culture de rente.

\section{Collecte des données}

Ce travail a été conduit à l'aide d'un questionnaire administré à 180 ménages répartis dans 12 villages de quatre communes (Aguié, Djirataoua, Guidan Roumdji et Sabon-Machi) de la région de Maradi. Le choix des communes a été guidé par les résultats d'une étude diagnostique réalisée par l'Institut National de la Recherche Agronomique du Niger (INRAN) et ses partenaires. Des missions terrain à travers la région de Maradi ont relevé des contraintes majeures des populations dans ces communes. Ces contraintes sont relatives à l'insécurité alimentaire et l'état de pauvreté extrême de la population de ces communes. Le faible accès aux moyens de production, surtout pour les femmes, limitent leurs possibilités de production. Le choix des villages a été fait suite aux entretiens mené avec les services techniques en rapport avec les objectifs de l'étude. Ainsi, au niveau de chaque village, des assemblées villageoises sont organisées et par la suite quinze, parmi les producteurs qui ont accepté de répondre individuellement au questionnaire, quinze (15) sont retenus de façon aléatoire. L'entretien a porté sur différents thèmes bien structurés, notamment l'identification de l'exploitant, le profil socioéconomique, la structure du troupeau, les objectifs de production, la conduite du troupeau, le système d'alimentation, la santé animale, ainsi que les principales contraintes d'élevage. Le Tableau 1 donne la répartition des producteurs par village.

\section{Analyses des données}

Les données collectées ont été codifiées et saisies sur le tableur Excel 2010, puis regroupées par catégories. L'analyse des données a été réalisée avec le logiciel Minitab version14 à travers le calcul des fréquences et des proportions pour les différentes variables.

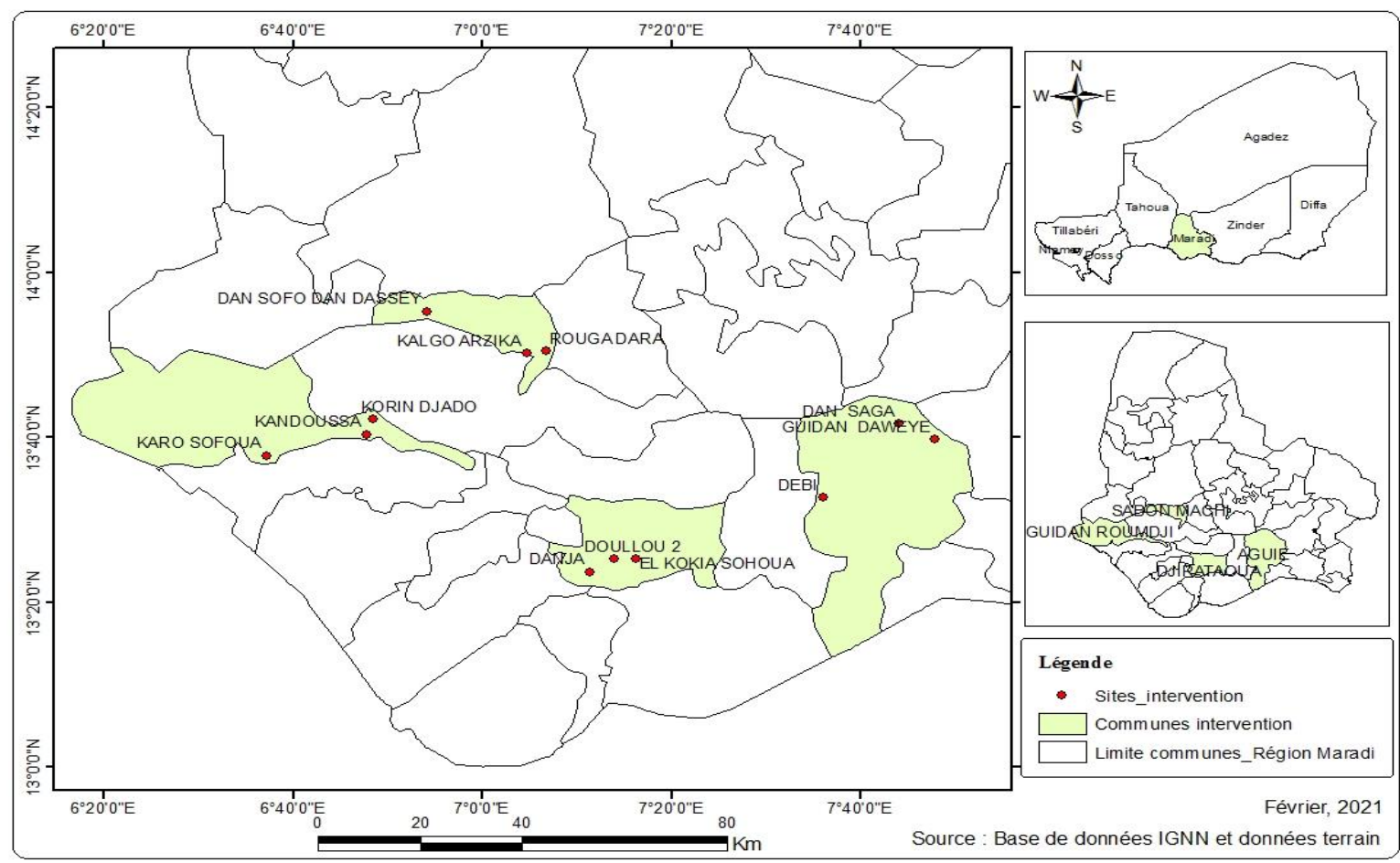

Figure 1 : Localisation des sites d'enquête. 
Tableau 1 : Répartition des éleveurs enquêtés par commune et par village.

\begin{tabular}{|c|c|c|c|c|}
\hline Communes & Villages & $\begin{array}{l}\text { Nombre de } \\
\text { enquêtés }\end{array}$ & personnes & $\begin{array}{l}\text { Nombres de têtes de } \\
\text { caprins détenus }\end{array}$ \\
\hline \multirow{3}{*}{ Sabon Machi } & Dan Dassey & 15 & & 71 \\
\hline & Kalgo Arzika & 15 & & 98 \\
\hline & Dara Ahmadou & 15 & & 83 \\
\hline \multirow[t]{3}{*}{ Aguié } & Dan Saga & 15 & & 68 \\
\hline & Guidan Daweye & 15 & & 66 \\
\hline & Maigaoudi & 15 & & 63 \\
\hline Guidan & Koren Jado & 15 & & 59 \\
\hline \multirow[t]{2}{*}{ Roumdji } & Karo Sofoua & 15 & & 91 \\
\hline & Kandoussa & 15 & & 80 \\
\hline \multirow[t]{3}{*}{ Djirataoua } & Danja & 15 & & 83 \\
\hline & Doulou & 15 & & 70 \\
\hline & El kokia & 15 & & 55 \\
\hline Total & 12 & 180 & & 887 \\
\hline
\end{tabular}

\section{RESULTATS}

\section{Caractéristiques socio-économiques des éleveurs}

Les caractéristiques des éleveurs enquêtés dans la zone d'étude sont présentées dans le Tableau 2. Les propriétaires des caprins sont en majorité des femmes de ménage $(72,8 \%)$ et d'ethnie haoussa $(93,9 \%)$. La tranche d'âge qui prédomine (36 à 50 ans) représente $(49,4 \%)$. Plus de $42 \%$ n'ont jamais été scolarisées, néanmoins $17,8 \%$ sont alphabétisées en langue arabe. La majorité des répondants vivent principalement des activités agricoles $(78,3 \%)$ et seuls $17,2 \%$ considèrent l'élevage comme une activité principale. Ces deux activités sont le plus souvent associées au petit commerce.

\section{Mode d'acquisition et objectifs de production de la chèvre rousse de Maradi}

Le Tableau 3 présente les différents modes d'acquisition des chèvres rousses recensés dans la zone d'étude. L'achat constitue le mode d'acquisition dominant avec $42,3 \%$; il est suivi par le "habbanayé" (prêt des animaux à un parent proche) et le don avec respectivement 25,1 et $19,7 \%$. Les principales motivations à élever la chèvre rousse sont l'épargne et la vente avec des proportions respectives de 41 et $36 \%$. Elles sont élevées principalement pour la production de lait par $22 \%$ des ménages enquêtés. Seul $1 \%$ des ménages les gardent en priorité pour la viande.

\section{Système de gestion des troupeaux}

Types d'habitats des élevages de la chèvre rousse de Maradi

Quarante-deux pour cent $(42,2 \%)$ des enquêtés ne disposent pas d'habitats pour leur troupeau caprin (Tableau 4). Ceux qui en disposent deux types de chèvrerie ont été distinguées, tous de types traditionnels. Les hangars confectionnés avec des toitures à base des tiges de céréales sont les plus répandus $(39,4 \%)$ dans la zone d'étude. Environ quatorze pour cent $(13,9 \%)$ dispose des enclos conçus en banco.

\section{Mode de conduite des caprins}

Le mode de conduite des animaux pratiqué varie selon la saison (Tableau 5). Le gardiennage avec berger domine $(78,4 \%)$ pendant la saison sèche. Les animaux du village sont regroupés le matin et conduit par un berger qui le ramène à midi pour l'abreuvement. L'après-midi, le troupeau retourne au pâturage jusqu'au soir. En saison de pluies la stabulation $(91,7 \%)$ est le mode de 
conduite le plus pratiqué. Les animaux sont mis au piquet dès l'installation des cultures jusqu'après les récoltes. La divagation est très peu $(2,2 \%)$ développée en cette saison des pluies, les animaux ne sortent pas du village et consomment l'herbe disponible aux alentours des concessions.

\section{Ressources fourragères et alimentation des animaux \\ Ressources fourragères}

Il ressort des résultats de l'enquête que pour les herbacées, Eragrostis tremula occupe le premier rang, suivi par Alysicarpus ovalifolius, Brachiara $s p$ et Jacquemontia tamnifolia. Quant au fourrage aérien utilisé, il est dominé surtout par Guera senegalensis, Piliostigma reticulatum, Sclérocarya birrea, Faidherbia albida et Ziziphus mauritiana (Tableau 6)

\section{Conduite de l'alimentation des caprins}

Les principales sources des compléments se composent des résidus de culture stockés, de la paille de brousse, des sous-produits agro-industriels et des minéraux (Figure 2). Les résidus de culture et le son local qui sont utilisés proviennent pour la plupart de l'exploitation. Le son local est donné sous forme de barbotage par une part importante $(87 \%)$ de l'échantillon enquêté. Les résidus de culture stockés comme les fanes d'arachide, les fanes et cosses de niébé sont distribués en saison sèche chaude comme compléments. La moitié des producteurs (51\%) font recours à l'achat du son de blé. La complémentation minérale est basée sur le sel de cuisine, le natron, la pierre à lécher et le sel de Bilma.

Plus de $70 \%$ des éleveurs ont déclaré complémenter tout leur troupeau (Figure 3) sans distinction ; 9,1\% complémentent uniquement les boucs destinés à la vente. Les chèvres suitées n'étaient complémentées que par 11,9\% des éleveurs.

La majorité $(64,4 \%)$ des éleveurs abreuve leurs animaux deux fois par jours $(64,4 \%)$, l'eau est servie le matin avant la conduite au pâturage puis le soir au retour.

\section{Gestion de la santé des animaux}

Le résultat montre qu'environ $58 \%$ des éleveurs vaccinent leurs animaux. Cependant, ceux qui font le déparasitage sont plus importants, soit $82,2 \%$ (Figure 4). Le recours aux services auxiliaires d'élevage est courant. La campagne de vaccination du cheptel commandité par l'Etat est une opportunité saisie par les éleveurs pour vacciner leurs animaux.

\section{Principales contraintes de l'élevage de la chèvre rousse de Maradi}

Les principales contraintes à la production caprine sont rapportées sur le Tableau 7. Il ressort de ce tableau que l'alimentation $(57,8 \%)$ constitue la contrainte majeure de l'élevage de la chèvre rousse de Maradi dans la zone d'étude. La plupart des éleveurs avancent comme causes, la raréfaction des ressources fourragères en saison sèche, la réduction des aires de pâture accentuée par la pression foncière et la difficulté d'accès aux intrants zootechniques, auxquelles s'ajoute l'absence des banques d'aliments bétail fonctionnelle de proximité. L'achat des sous-produits agro industriels se fait en détail avec l'acquisition des quelques mesures le jour du marché hebdomadaire. Après le problème d'alimentation, vient la contrainte des maladies animales avec une proportion de 17,2\%. Les parasitoses, les diarrhées et la peste des petits ruminants sont les principales pathologies les plus signalées. Quant à la stabulation, elle a été citée comme cause du faible niveau de production par 7,8\% de l'échantillon d'enquête. Durant la saison des pluies, les caprins sont gardés en stabulation entravée dans des conditions précaires exposés aux intempéries. Les vols d'animaux, le problème de logement, le manque de berger et l'absence de boucs reproducteurs ont été moins rapportés, avec des proportions inférieures à $3 \%$ chacun. 
Tableau 2 : Caractéristiques socio-économiques des éleveurs de chèvres rousses au niveau de la région de Maradi au Niger ( $\mathrm{n}=180)$.

\begin{tabular}{|c|c|c|}
\hline Variables & Nombre & Pourcentage (\%) \\
\hline \multicolumn{3}{|l|}{ Sexe } \\
\hline Homme & 49 & 27,2 \\
\hline Femme & 131 & 72,8 \\
\hline Total & 180 & 100 \\
\hline \multicolumn{3}{|l|}{ Age } \\
\hline$<$ à 18 ans & 3 & 1,7 \\
\hline 18 à 35 ans & 77 & 42,8 \\
\hline 36 à 50 ans & 89 & 49,4 \\
\hline$>50$ & 11 & 6,1 \\
\hline Total & 180 & 100 \\
\hline \multicolumn{3}{|c|}{ Statut matrimonial } \\
\hline Célibataire & 16 & 8,9 \\
\hline Marié (e) & 154 & 85,6 \\
\hline Veuf/veuve & 8 & 4,4 \\
\hline Divorcé (e) & 2 & 1,1 \\
\hline Total & 180 & 100 \\
\hline \multicolumn{3}{|l|}{ Ethnie } \\
\hline Haoussa & 169 & 93,9 \\
\hline Peulh & 4 & 2,2 \\
\hline Touareg & 7 & 3,9 \\
\hline Total & 180 & 100 \\
\hline \multicolumn{3}{|c|}{ Niveau d'instruction } \\
\hline Analphabète & 76 & 42,2 \\
\hline Primaire & 39 & 21,7 \\
\hline Secondaire & 32 & 17,8 \\
\hline Supérieur & 1 & 0,6 \\
\hline Alphabétisé & 32 & 17,8 \\
\hline Total & 180 & 100 \\
\hline \multicolumn{3}{|c|}{ Activité principale } \\
\hline Agriculture & 141 & 78,3 \\
\hline Elevage & 30 & 16,7 \\
\hline Commerce & 7 & 3,9 \\
\hline Autres & 2 & 1,1 \\
\hline Total & 180 & 100 \\
\hline \multicolumn{3}{|c|}{ Activité secondaire } \\
\hline Agriculture & 18 & 10,0 \\
\hline Elevage & 129 & 71,7 \\
\hline Commerce & 29 & 16,1 \\
\hline Autres & 4 & 2,2 \\
\hline Total & 180 & 100 \\
\hline
\end{tabular}


Tableau 3 : Modes d'acquisition et objectifs de production des caprins dans la zone d'étude.

\begin{tabular}{lcc}
\hline Mode d'acquisition & Nombre d'éleveurs & \% éleveurs \\
\hline Héritage & 18 & 10,1 \\
Don & 35 & 19,7 \\
Achat & 76 & 42,3 \\
Habbanayé & 45 & 25,1 \\
Confiage & 5 & 2,8 \\
Total & 180 & 100 \\
\hline Objectifs de production & Nombre d'éleveurs & \% éleveurs \\
\hline Epargne & 77 & 43 \\
Vente & 61 & 34 \\
Lait & 40 & 22 \\
Viande & 2 & 1 \\
Total & 180 & 100 \\
\hline
\end{tabular}

Tableau 4 : Types de logement des chèvres rousses dans la région de Maradi.

\begin{tabular}{lcc}
\hline Types habitat & Nombre & Pourcentage (\%) \\
\hline Pas habitat & 76 & 42,2 \\
Enclos & 25 & 13,9 \\
Habitat couvert en matériaux & 71 & 39,4 \\
non définitifs & & 100 \\
\hline Total & 180 & \\
\hline
\end{tabular}

Tableau 5 : Différents modes de conduite des caprins dans la région de Maradi au Niger.

\begin{tabular}{lcccc}
\hline Conduite & \multicolumn{2}{c}{ Saison sèche } & \multicolumn{2}{c}{ Saison de pluies } \\
\hline Divagation & Nombre & \% & Nombre & $\%$ \\
Gardiennage & 39 & 21,7 & 4 & 2,2 \\
avec berger & 133 & 73,9 & 23 & 7,2 \\
Stabulation & 8 & 4,4 & 147 & 91,7 \\
Total & 180 & 100 & 180 & 100,0 \\
\hline
\end{tabular}

Tableau 6 : Principales espèces fourragères pâturées par les caprins.

\begin{tabular}{|c|c|c|c|c|}
\hline $\begin{array}{l}\text { Types } \\
\text { fourrages }\end{array}$ & de & $\begin{array}{l}\text { Noms } \\
\text { vernaculaires }\end{array}$ & Noms scientifiques & $\begin{array}{l}\text { Fréquence } \\
\text { des espèces } \\
\text { appétées }\end{array}$ \\
\hline \multirow[t]{8}{*}{ Herbacées } & & Cinciya & Eragrostis tremula & 106 \\
\hline & & Gadagui & Alysicarpus ovalifoluis & 48 \\
\hline & & Gamba & Andropogon gayanus & 25 \\
\hline & & Garaji & Brachiara sp & 16 \\
\hline & & Kunkum bara & Jacquemontia tamnifolia & 14 \\
\hline & & Alkaman'turuwa & Borreria scabra & 11 \\
\hline & & Yalyadi & Ipomea vagans & 11 \\
\hline & & Tsap & Phyllanthus pentandrus & 8 \\
\hline
\end{tabular}


M. AZIADA MOLID et al. / Int. J. Biol. Chem. Sci. 15(3): 936-949, 2021

\begin{tabular}{llll}
\hline Ligneux & Sabara & Guera senegalensis & 145 \\
Kalgo & Piliostigma reticulatum & 69 \\
Magaria & Ziziphis mauritiana & 33 \\
Gao & Faidherbia albida & 27 \\
& Danya & Sclérocarya birrea & 29 \\
D'un'du & Dichrastachys cinera & 16 \\
Sark'aky'a & Accacia ataxacantha & 13 \\
\hline
\end{tabular}

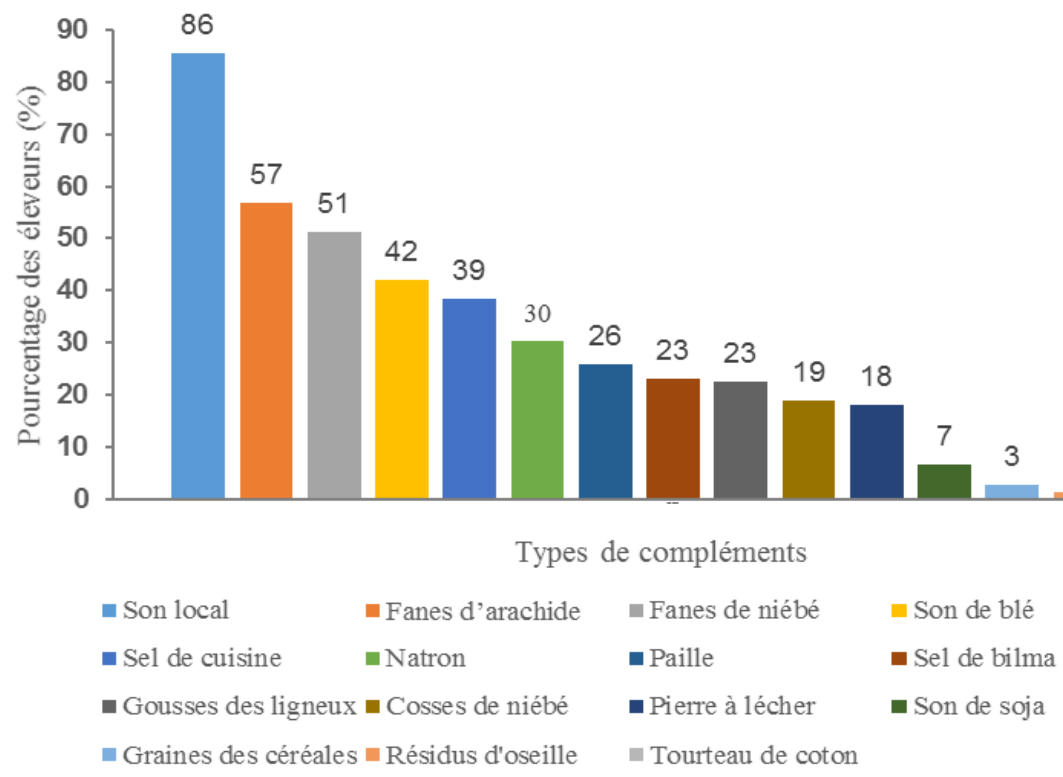

Figure 2 : Principaux compléments alimentaires utilisés par les éleveurs enquêtés.

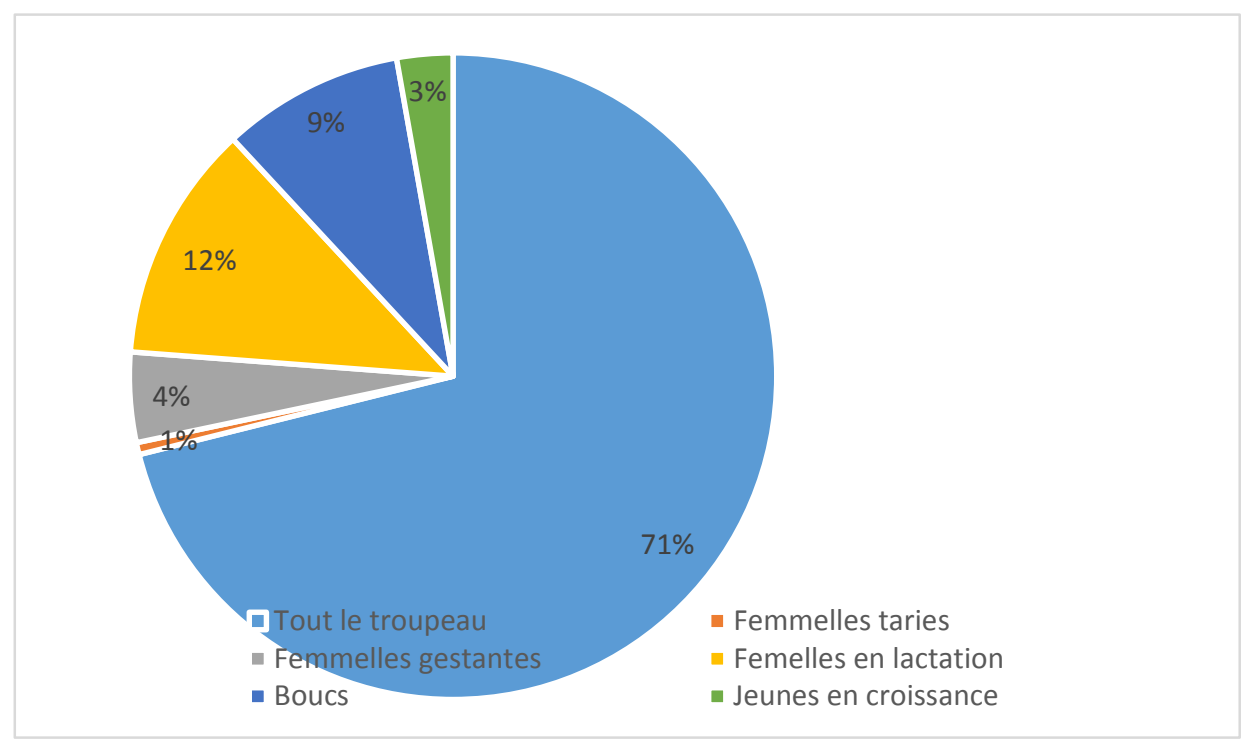

Figure 3 : Catégories d'animaux complémentés par les éleveurs de la chèvre rousse. 


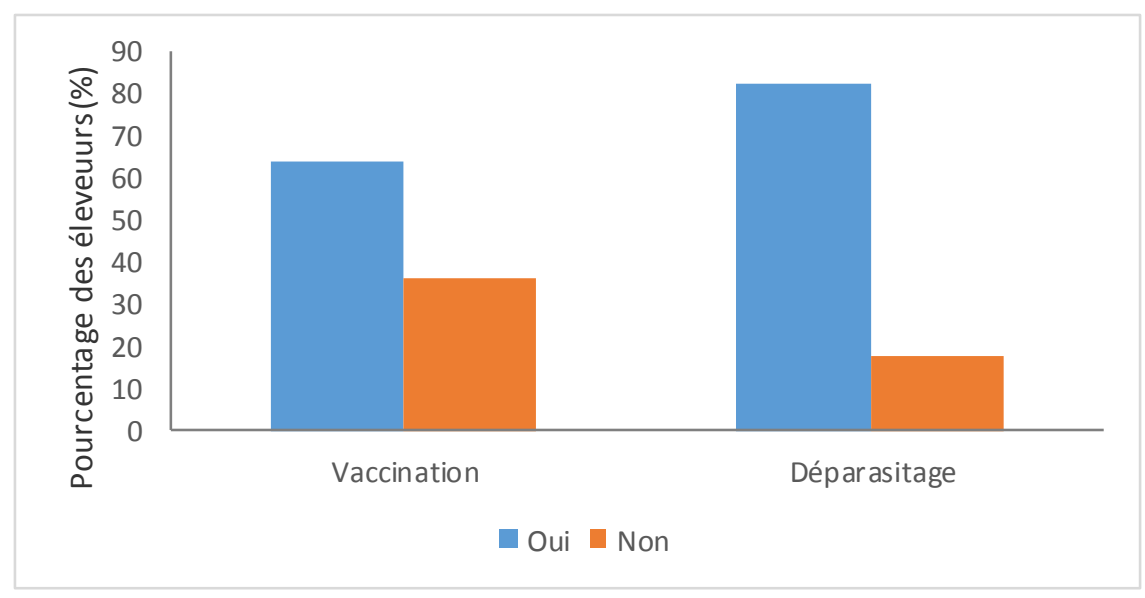

Figure 4 : Pratiques de la vaccination et du déparasitage.

Tableau 7 : Principales contraintes de l'élevage de la chèvre rousse dans la région de Maradi.

\begin{tabular}{|c|c|c|}
\hline Contraintes & Fréquence des répondants & Proportion (\%) \\
\hline Pénurie d'aliments & 104 & 57,8 \\
\hline $\begin{array}{l}\text { Faible accès aux sous-produits } \\
\text { agro industriels }\end{array}$ & 11 & 6,1 \\
\hline logement inadéquat & 6 & 3,3 \\
\hline Santé animale & 31 & 17,2 \\
\hline Manque de berger & 4 & 2,2 \\
\hline absence des boucs & 5 & 2,8 \\
\hline Vols d'animaux & 5 & 2,8 \\
\hline $\begin{array}{l}\text { Stabulation en saison des } \\
\text { pluies }\end{array}$ & 14 & 7,8 \\
\hline Total & 180 & 100 \\
\hline
\end{tabular}

\section{DISCUSSION}

\section{Caractéristiques socio-économiques des éleveurs}

Les résultats de cette étude ont montré que l'élevage de la chèvre rousse est une activité pratiquée par les femmes $(72,8 \%)$ en majorité mariées. Selon Hassan et al. (2019) un fort taux des mariés chez les enquêtés traduirait le niveau de responsabilité sociale et l'expérience des éleveurs dans la gestion et la conduite des animaux. Le faible nombre d'hommes propriétaires des caprins a été observé par les études précédentes rapportées par Saadou (2005) et Karimou (2015). La variation de sexe dans la possession des animaux a montré que les femmes ont toujours possédé la plus grande proportion des chèvres dans les ménages ruraux (Ejlertsen et al., 2013). En outre, les chefs d'exploitation sont connus pour être détenteurs des gros ruminants du ménage. Selon les hommes interviewés dans cette étude, en cas de besoin du ménage, leur part du troupeau caprin est la première à être vendue.

Seul $16,7 \%$ des personnes considèrent l'élevage comme activité principale. La production agricole étant la principale source des revenus des ménages. L'intégration agriculture élevage est très développée dans le centre sud du Niger qui fournit la première production agricole du pays. Le petit commerce est pratiqué comme activité secondaire par $16,1 \%$ des personnes. Dans la même région, Amole et Ayantunde (2016) ont 
signalé que les autres activités commerciales et de travail en dehors du village ont contribué respectivement à 26 et $24 \%$ au revenu du ménage, ce qui est plus que la production animale.

\section{Mode d'acquisition et objectifs de production de la chèvre rousse}

La proportion des femmes ayant acquis leurs animaux par achat $(45,8 \%)$ illustre bien l'intérêt qu'elles accordent à cette activité. Les femmes s'associent à travers les pratiques d'épargne communautaire appelée tontine. A tour de rôle chaque membre reçoit une somme d'argent fixée dès le départ par consensus. Ce fond collecté est le plus souvent utilisé pour l'acquisition d'une ou de plusieurs chèvres. Le "Habbanayé" représente le deuxième mode d'acquisition des animaux après l'achat. Le "Habbanayé" est une pratique d'entre aide sociale consistant à donner quelques têtes d'animaux à un parent proche qui les élève afin que les descendants lui appartiennent. Cette pratique très développée chez les communautés peulhs a été adaptée par les projets intervenant dans la lutte contre l'insécurité alimentaire. Ainsi, des groupements féminins sont appuyés avec des kits composés de quatre (4) chèvres et un bouc plus un sac $50 \mathrm{~kg}$ d'aliments pour bétail. Chaque membre du groupe garde les animaux pendant une période de 12 à 18 mois. La femme qui reçoit les chèvres en prêt assume la responsabilité jusqu'au sevrage des chevreaux.

Les chèvres sont élevées principalement pour l'épargne, pour la vente et le lait. Cependant, dans la plupart des cas, le lait est destiné à la consommation des chevreaux pour leur bonne croissance. L'élevage caprin constitue une source de revenus pour les ménages, en particulier pour les femmes, à travers la vente d'animaux sur pied, le lait et les produits laitiers (Missohou et al., 2004). Seul $1 \%$ des éleveurs gardent les animaux pour la viande. Nos résultats sont différents de plusieurs études menées sur les caprins comme ceux de Ajala et al. (2004); Missohou et al. (2016). Au Nigeria, Alikwe et al. (2011) ont rapporté que la viande est la principale forme de consommation des caprins. Au Benin, Idrissou et al. (2018), ont rapporté qu'en zone soudanienne la chèvre naine est principalement utilisée pour la production de viande. Les différences observées pourraient être dues au fait qu'en milieu sahélien la viande des ovins est plus appréciée par rapport à celle des caprins. Le choix de l'élevage de la chèvre rousse est aussi motivé pour sa prolificité et pour la qualité de sa peau. Ce qui lui a valu une attention particulière et a incité plusieurs actions au niveau national permettant sa diffusion (Marichatou et al., 2002).

\section{Habitat et mode de conduite des animaux}

Dans notre étude $42,2 \%$ des producteurs ne disposent pas de logement pour leurs animaux, seul 13,9\% ont construit des hangars avec du bois et des tiges de céréales. Les éleveurs ignorent le rôle du logement sur les performances de production des animaux. D'où la nécessité de renforcer leurs capacités sur les bonnes pratiques d'élevage. Pendant la saison de pluies en absence des jachères la stabulation constitue le mode de conduite dominant. Ce résultat est conforme à celui de Ali et al. (2003) qui ont souligné que la stabulation est le mode de conduite le plus rependu en saison de pluies en zone péri urbaine de Maradi. Durant cette période, l'alimentation se fait à l'auge avec du fourrage vert récolté au champ. En saison sèche la majorité des éleveurs sollicitent le service d'un berger pour la conduite des animaux au pâturage. Ce qui a été déjà démontré par Ali et al. (2003) où le gardiennage était pratiqué en saison sèche pour permettre aux animaux de s'épanouir et profiter des résidus de culture laissés dans le champ. Selon Hassan et al. (2019), chez les éleveurs haoussas, la conduite du troupeau est en grande partie salariale contrairement aux Peulhs et Touaregs, chez qui la main d'œuvre est familiale. Le règlement du berger est hebdomadaire et peut se faire en nature comme en espère. Le choix de ce mode de conduite est motivé surtout par le vol récurrent du bétail dans cette zone. 


\section{Ressources fourragères et conduite de l'alimentation}

Les ressources fourragères les plus exploitées sont constituées d'herbacées vivaces et pérennes, des feuilles et gousses des ligneux fourragers. Les principales espèces fourragères identifiées dans cette étude sont en accord avec celles rapportées par Dan Gomma et al. (2017). Pendant la saison sèche, les parcours naturels et les résidus de cultures disponibles pour le bétail après la récolte sont généralement fibreux et dépourvues de la plupart des nutriments essentiels. Pour compenser les pertes de poids et réduire le risque de mortalité, les éleveurs font un apport de complément alimentaire. Les principales ressources utilisées proviennent pour la plupart de l'exploitation, elles se composent des résidus de récolte, du son local et de la paille de brousse. Cette observation est similaire à celle de Zoundi et al. (2003) au Burkina Faso. Le son de blé est le principal concentré acheté par les éleveurs au marché, dont le tiers de l'échantillon dit avoir fait recours à son achat. Ce résultat est en accord avec celui rapporté par Amole et Ayantunde (2016). Les résidus de culture stockés sont distribués aux animaux en saison sèche quand les pâturages deviennent rares. On assiste souvent à un gaspillage de ces ressources pendant la période poste récolte, au moment où le stock est assez abondant. Les éleveurs ont tendance à les distribuer aux animaux sans tenir compte de la longue période sèche. Cela entraine le plus souvent, l'épuisement du stock avant la fin de la période de soudure. La complémentation avec le son local était pratiquée par la majorité des éleveurs. Cela se justifie par le fait qu'il est le sous-produit des principales céréales (mil et sorgho) consommées par les ménages ruraux. Très peu d'éleveurs ont recours $(1,3 \%)$ à la complémentation avec le tourteau de coton. Les sous-produits agro-industriels en général et le tourteau des graines de coton en particulier sont plus distribués aux bovins et aux ovins en embouche. Notre résultat est différent de celui de Lawal et al. (2018) qui ont trouvé en zone péri urbaine et urbaine de Niamey des proportions respectives de 42,6 à
65,4\% des éleveurs utilisant les sous-produits agro-industriels. Cette différence pourrait être liée au fait que leur étude a eu lieu en milieu sédentaire où la pratique de l'élevage est plus intensifiée. Les caprins reçoivent moins de complémentation que les ovins, les éleveurs les juges plus rustiques et peut-être de moindre valeur (Missohou et al., 2016). Diverses sources des minéraux sont utilisées pour la supplémentation des caprins, notamment le sel de cuisine et celui de Bilma, les pierres à lécher et le natron. Ce résultat est conforme à celui de Ali et al. (2003), qui ont rapporté l'utilisation fréquente du sel de cuisine et de Bilma en saison de pluies. Ces suppléments minéraux sont généralement disponibles sur le marché local. La majorité des éleveurs ont déclaré que la supplémentation à base de natron favorise la consommation d'eau chez les animaux. La complémentation ne prend pas en compte les besoins des animaux, elle est surtout fonction de la disponibilité des ressources alimentaires stockées. Selon Alexendre et al. (2012) dans les régions tropicales, les agriculteurs sont davantage enclins à gérer l'alimentation de leur troupeau en fonction des ressources alimentaires disponibles plutôt qu'en fonction des besoins des animaux. Beaucoup d'éleveurs interviewés conçoivent mal une complémentation ciblée destinée à une catégorie d'animaux. Ce qui explique la faible proportion des éleveurs qui font une complémentation destinée aux femelles en lactation. Une étude menée au Burkina par Zoundi et al. (2003) a montré les stratégies de complémentation des femelles en lactation semblent calqués sur l'évolution saisonnière des disponibilités alimentaires. Il est nécessaire d'adapter les stratégies de complémentation afin de garantir une gestion optimale des ressources alimentaires.

\section{Contraintes limitant la productivité de l'élevage de la chèvre rousse}

La faible disponibilité des ressources alimentaires est apparue comme la contrainte majeure à la production caprine. Environ 58\% des éleveurs affirme que les difficultés à nourrir leur troupeau toute l'année constitue 
leur principal défi. Les raisons avancées sont entre autre, la rareté des fourrages en saison sèche, la forte pression foncière qui a réduit les espaces pâturables pour le bétail et la pauvreté des résidus de récolte. Ceci est en accord avec le constat des plusieurs études menées au sahel par Ali et al. (2003) ; Zoundi et al. (2003). Dans les systèmes mixtes, les résidus de culture jouent un rôle important dans l'alimentation du bétail. Cependant, ils sont caractérisés par une faible teneur en éléments nutritifs, une teneur élevée en fibre, une faible digestibilité et une faible consommation volontaire (Rumosa Gwaze et al., 2009). Les maladies animales sont le second problème qui a été évoqué. Les parasitoses, les diarrhées, les infections respiratoires ont été les plus évoquées. Dans les pays en développement comme le Niger, les petits producteurs ont généralement un accès limité aux médicaments pour la santé de leur bétail. La stabulation en saison de pluies a été incriminée comme une condition non confortable par $7,8 \%$ des enquêtés. Durant cette longue période, les petits ruminants sont mis en claustration et nourrit avec l'herbe fauchée. Ce qui le prédispose au déficit nutritionnel et aux maladies lorsque les apports sont insuffisants. Awa et al. (2004) ont rapporté des taux de mortalité liée au confinement des petits ruminants de l'ordre de 15 à 60\%. Comme l'alimentation est restreinte, les chèvres ont peu de choix en matière d'alimentation, ce qui se traduit par une mauvaise condition physique (Assan et al., 2015). Selon $6 \%$ des producteurs le faible pouvoir d'achat limite l'accès aux sousproduits agro industriels en saison sèche chaude, coïncidant avec l'épuisement du stock alimentaire du ménage. Durant cette période les animaux sont fatigués et leur valeur marchande chute. Aussi, la satisfaction du besoin du ménage passe en priorité. Quant à la stabulation entravée en saison de pluie, elle induit le stress nutritionnel et des problèmes sanitaires liés à l'hygiène de l'habitat. Tous ces facteurs ont des répercussions sur la productivité des animaux. Une étude menée au Benin par Dossa et al. (2007) a fait ressortir que les principales contraintes à l'élevage des petits ruminants sont surtout d'ordre sanitaire. Au Botswana, Monau et al. (2017) ont rapporté que les principales contraintes à la production caprine sont les prédateurs et les vols d'animaux.

\section{Conclusion}

Cette étude visait à caractériser les pratiques d'élevage et à cerner les contraintes à la production caprine. L'élevage de la chèvre rousse participe à l'autonomisation des femmes, et à la résilience d'une manière générale des producteurs. Les caprins étaient élevées principalement pour l'épargne, pour la vente et pour la production laitière. La pénurie des aliments, les maladies animales et la stabulation en saison de pluie s'avèrent être les principaux défis auxquels font face les éleveurs. La pratique de la complémentation ciblée est peu développée et dépend du disponible en ressources alimentaires. La majorité des éleveurs complémentent tout leur troupeau. En saison de pluies, la complémentation minérale se fait principalement avec le sel de cuisine et le natron. Les renforcements des capacités des producteurs sur les techniques de complémentation adaptées pourraient booster la productivité de manière générale.

\section{CONFLITS D'INTERETS}

Les auteurs de ce manuscrit déclarent qu'il n'y'a aucun conflit d'intérêt entre eux.

\section{CONTRIBUTIONS DES AUTEURS}

Dans le cadre de cette étude, MMA, a assuré l'élaboration du protocole, la collecte et le traitement des données, la rédaction du premier manuscrit sous l'encadrement de MC et de SI. OMMM a contribué à l'élaboration des fiches d'enquête et à la relecture des différentes versions pour l'amélioration de la qualité scientifique du manuscrit.

\section{REMERCIEMENTS}

Nos remerciements vont également à l'Institut National de la Recherche Agronomique du Niger (INRAN) et aux agents communaux du service d'élevage des 
quatre communes d'intervention pour l'appui technique.

\section{REFERENCES}

Abdou MM, Issa S, Gomma AD, Sawadogo GJ. 2017. Analyse technico-économique des Aliments densifies sur les performances de croissances des boucs roux de Maradi au Niger. Int. J. Bio. Chem. Sci., 11(1): 280-291. DOI: https://doi.org/10.4314/ijbcs.v11i1.22

Abdou N, Gouro AS, Nsahlai IV. 2018. Effect of feeding level on production performance of lactating Red Maradi goats and post-natal performance of kids. Int. J. Bio. Chem. Sci., 11(6): 2826-2836. DOI: https://doi.org/10.4314/ijbcs.v11i6.21

Ajala M., Lamidi O, Otaru S. 2008. Periurban small ruminant production in Northern Guinea Savanna, Nigeria. Asian J. Anim. Vet. Adv., 3(3): 138-146. DOI: 10.3923/ajava.2008.138.146

Alexandre G, Arquet R, Fleury J, Troupé W, Boval M, Archimède $\mathrm{H}$, Mahieu $\mathrm{M}$, Mandonnet N. 2012. Systèmes d'élevage caprins en zone tropicale: analyse des fonctions et des performances. INRA Prod. Anim., 25 : 305-316. DOI: https://doi.org/10.20870/productionsanimales.2012.25.3.3218

Alexandre G, Leimbacher F, Maurice O, Domarin D, Naves M, Mandonnet N. 2009. Goat farming systems in Martinique: management and breeding strategies. Trop Anim Health Prod., 41: 635-644.

DOI:

https://doi.org/10.1007/s11250-0089235-1

Ali L, Van den Bossche P, Thys E. 2003. Enjeux et contraintes de l'élevage urbain et périurbain des petits ruminants à Maradi au Niger : quel avenir ? Revue Élev. Méd. Vét. Pays. Trop., 56(1-2): 73-82.

DOI:

https://doi.org/10.19182/remvt.9879

Alikwe PCN, Faremi AY, Fajemisin AN, Akinsoyinu AO. 2011. Performances and nitrogen utilization of West African Dwarf goats fed soybean and dried poultry waste-based concentrates as supplements to cynodon nlemfuensis basal diet. Journal of Applied Sciences in Environmental Sanitation, 6(2): 191189.

Amole TA, Ayantunde AA. 2016. Assessment of Existing and Potential Feed Resources for Improving Livestock Productivity in Niger. International J. of Agricultural Research, 11: 40-55. DOI: https://doi.org/10.3923/ijar.2016.40.55

Assan N. 2015. Effect of Nutrition on Yield and Milk Composition in Sheep and Goats. Scientific Journal of Animal Science, 4(1): 1-10. DOI: http://dx.doi.org/10.14196/sjas.v4i1.1806

Barazi AA, Chaibou M, Mahaman MMO, Nouhou ME. 2019. Effet de la supplémentation des rations par un additif alimentaire sur la croissance pondérale et le rendement carcasse des chevreaux Roux de Maradi au Niger. Int. J. Biol. Chem. Sci., 13(6): 2487-2496. DOI: https://dx.doi.org/10.4314/ijbcs.v13i6.5

Dan Gomma A, Chaibou I, Banoin M, Schlecht E. 2017. Commercialisation et valeur nutritive des fourrages dans les centres urbains au Niger : cas des villes de Maradi et de Niamey. International Journal of Innovation and Applied Studies, 21(3): 508-521.

Djibrillou OA, Pandey VS, Gouro SA, A Verhulst A. 1998. Effect of urea-treated or untreated straw with cotton seed on performances of lactating maradi (Red Sokoto) Goats in Niger. Livestock Production Science, 55(2): 117-25. DOI: $\quad$ https://doi.org/10.1016/S03016226 (98)00130-4

Dossa L, Wollny HC, Gauly M. 2007. Smallholders' perceptions of goat farming in Southern Benin and opportunities for improvement. Tropical Animal Health and Production, 39(1): 49 57. DOI: https://doi.org/10.1007/s11250006-4440-2

Ejlertsen M, Poole J, Marshall K. 2012. Traditional breeding objectives and practices of goat, sheep and cattle smallholders in The Gambia and implications in relation to the design of breeding interventions. Trop. Anim. 
Health Prod., 45: 219-229. DOI: https://doi.org/10.1007/s11250-0120194-1

Hassan YA, Guiguigbaza-Kossigan D, Moumouni I, Mamman M, Ibrahim I, Hamani M. 2019. Etude des pratiques d'élevage des moutons Peulh du Niger : le Peulh blanc et le Peulh bicolore. Int. J. Biol. Chem. Sci., 13(1): 83-98. DOI: https://dx.doi.org/10.4314/ijbcs.v13i1.8

FAO. 2013. Le développement du secteur de l'élevage pour la réduction de la pauvreté : perspectives économique et politique. FAO, Les nombreuses vertus de l'élevage, Rome. http://www.fao.org/3/i2744f/i2744f.pdf

FAO.2017. Revue des filières bétail/viande et de lait et des politiques qui les influencent au Niger, www.fao.org/3/ai5271f.pdf

Institut National de la Statistique. 2006. Questionnaires des indicateurs de base de bien être (QUIBB_2005), profil de pauvreté. INS, rapport d'analyse, Maradi, $69 \mathrm{p}$.

Institut National de la Statistique. 2009. Rapport régional sur les progrès vers l'atteinte des objectifs du millénaire à Maradi. INS, rapport d'analyse, Maradi, $72 \mathrm{p}$.

Karimou BH. 2015. Caractérisation phénotypique et zootechnique de la chèvre rousse de Maradi. Thèse de Doctorat en Productions Animales, Université Abdou Moumouni de Niamey, Niamey, 138 p.

Lawal AM, Chaibou M, Mani M, Garba M, Gouro A. 2018. Pratiques d'éleveurs et résultats économiques d'élevage dans les exploitations urbaines et périurbaines de Niamey. Int. J. Biol. Chem. Sci., 12(1): 294-309.

DOI: https://dx.doi.org/10.4314/ijbcs.v12i1.24

Laouali A. 2014. Contribution à l'étude de la dynamique de l'élevage pastoral au Niger : cas de la région de Diffa. Thèse de Doctorat, Gembloux Agro-Bio Tech., Université de Liège, Liège, 189 p.
Marichatou H, Mamane L, Banoin M, Baril G. 2002. Performances zootechniques des caprins au Niger : étude comparative de la chèvre rousse de Maradi et de la chèvre à robe noire dans la zone de Maradi. Revue Elev. Méd. Vét. des pays tropicaux, 55(1): 79-84.

Missohou A, Nahimana G, Ayssiwede SB, Sembene M. 2016. Elevage caprin en Afrique de l'Ouest: une synthèse. Rev. Elev. Med. Vet. Pays Trop., 69(3). DOI: https://doi.org/10.19182/remvt.31167

Monau PI, Visser C, Nsoso SJ, Van MarleKöster E. 2017. A survey analysis of indigenous goat production in communal farming systems of Botswana. Trop. Anim. Health Prod., 49: 1265-1271. DOI: https://doi.org/10.1007/s11250017-1324-6

Rumosa Gwaze F, Chimonyo M, Dzama K. 2009. Communal goat production in Southern Africa: a review. Trop. Anim. Health Prod., 41: 1157-1168. DOI: https://doi.org/10.1007/s11250-0089296-1

Saâdou M. 2005. Performances de reproduction et de production de la chèvre rousse de Maradi en milieu rural au Niger. Thèse de Méd. Vétérinaire, Dakar, Sénégal, $82 \mathrm{p}$.

Tolera A, Merkel RC, Goetsch AL, Sahlu T, Negesse T. 2000. Nutritional constraints and future prospects for goat production in East Africa. In The Opportunities and Challenges of Enhancing Goat Production in East Africa, Merkel RC, Abebe G, Goetsch AL (eds). Langston University press: Langston; 43-57.

Zoundi JS, Sawadogo L, Nianogo AJ. 2003. Pratiques et stratégies paysannes en matière de complémentation des ruminants au sein des systèmes d'exploitation mixte agriculture-élevage du plateau central et du Nord du Burkina Faso. Tropicultura, 21(3): 122-128. 